ACCEPTED MANUSCRIPT • OPEN ACCESS

\title{
A practical EPR dosimetry system for routine use in radiotherapy: uncertainty analysis of lithium formate dosimeters at the therapeutic dose level
}

To cite this article before publication: Sebastian Höfel et al 2020 Phys. Med. Biol. in press https://doi.org/10.1088/1361-6560/ab9414

\section{Manuscript version: Accepted Manuscript}

Accepted Manuscript is "the version of the article accepted for publication including all changes made as a result of the peer review process, and which may also include the addition to the article by IOP Publishing of a header, an article ID, a cover sheet and/or an 'Accepted Manuscript' watermark, but excluding any other editing, typesetting or other changes made by IOP Publishing and/or its licensors"

This Accepted Manuscript is (C) 2020 Institute of Physics and Engineering in Medicine.

As the Version of Record of this article is going to be / has been published on a gold open access basis under a CC BY 3.0 licence, this Accepted Manuscript is available for reuse under a CC BY 3.0 licence immediately.

Everyone is permitted to use all or part of the original content in this article, provided that they adhere to all the terms of the licence https://creativecommons.org/licences/by/3.0

Although reasonable endeavours have been taken to obtain all necessary permissions from third parties to include their copyrighted content within this article, their full citation and copyright line may not be present in this Accepted Manuscript version. Before using any content from this article, please refer to the Version of Record on IOPscience once published for full citation and copyright details, as permissions may be required. All third party content is fully copyright protected and is not published on a gold open access basis under a CC BY licence, unless that is specifically stated in the figure caption in the Version of Record.

View the article online for updates and enhancements. 


\section{A practical EPR dosimetry system for routine}

\section{use in radiotherapy: Uncertainty analysis of}

\section{lithium formate dosimeters at the therapeutic}

\section{dose level}

Sebastian Höfel ${ }^{1,2}$, Michael Stehle ${ }^{1}$, Felix Zwicker², Michael K. Fix ${ }^{3}$, Malte Drescher ${ }^{1}$

${ }^{1}$ Department of Chemistry and Konstanz Research School Chemical Biology, University of Konstanz,

Germany

${ }^{2}$ Klinik und Praxis für Strahlentherapie am Klinikum Konstanz, Konstanz, Germany

${ }^{3}$ Division of Medical Radiation Physics and Department of Radiation Oncology, Inselspital, Bern University Hospital and University of Bern, Switzerland

Address all correspondence to:

Prof. Dr. Malte Drescher Department of Chemistry University of Konstanz Box 706, Universitätsstraße 10

78457 Konstanz, Germany Phone: +49 $753188-5262$

Email: malte.drescher@uni-konstanz.de 


\section{Abstract}

In electron paramagnetic resonance (EPR) dosimetry, solid dosimeter materials such as alanine (AL) or, more recently, lithium formate monohydrate (LFM) are typically used. These materials offer high potential for applications in radiotherapy based on their favorable dosimetric properties. Nevertheless, EPR dosimetry is not widespread in the clinics. This work presents an uncertainty analysis of EPR dosimetry in the dose range from 1 to 70 Gy using a compact spectrometer and applying a practical procedure being suitable for routine use in radiotherapy. The performances of self-pressed LFM pellets and commercial AL pellets are compared side by side.

All pellets had a diameter of $4 \mathrm{~mm}$ and a height of $2 \mathrm{~mm}$ (AL) or $4 \mathrm{~mm}$ (LFM). The mean pellet mass was $35.81 \mathrm{mg}$ and $73.81 \mathrm{mg}$ for AL and LFM, respectively. Before irradiation, the pellets were stored for at least 8 weeks at $34 \pm 2 \%$ relative humidity. For irradiation, the pellets were put inside an airtight capsule. In total, 25 pellets per material were examined. The pellets were irradiated at a temperature of $25 \pm 2.5(2 \sigma){ }^{\circ} \mathrm{C}$ to doses of either 1, 5, 20, 50 or $70 \mathrm{~Gy}$ (five pellets per dose value and material) by a clinical $6 \mathrm{MV}$ photon beam. Measurement uncertainties were obtained from five independent readouts per pellet within five weeks following irradiation using a benchtop EPR spectrometer. The measurement time of a single readout was restricted to $10 \mathrm{~min}$ per pellet. Dose values were derived from EPR signal amplitudes using a specifically developed spectral fitting procedure. Signal fading characteristics were analyzed and taken into account during evaluation.

The relative dose uncertainties $(1 \sigma)$ for a single readout at doses $\geq 5$ Gy are below $2.8 \%$ (AL) and $1.1 \%$ (LFM) but increase to $12.3 \%(\mathrm{AL})$ and $2.6 \%$ (LFM) at $1 \mathrm{~Gy}$. By averaging five independent readouts, the uncertainties at $1 \mathrm{~Gy}$ decrease to $2.6 \%$ (AL) and $0.8 \%$ (LFM).

In terms of dose uncertainty, the LFM pellets are superior to the commercial AL pellets owing to their narrower EPR spectrum and approximately doubled mass resulting in higher EPR signal intensities. In case of the LFM pellets, the EPR dosimetry system shows a high level of precision (<3\%) down to 1 Gy being preferable for applications in radiotherapy. The uncertainties can be further decreased by averaging multiple dose values from independent readouts.

Keywords: EPR dosimetry, alanine, lithium formate, radiotherapy, uncertainty 


\section{Introduction}

Electron paramagnetic resonance (EPR) spectroscopy is a specific method for the detection of unpaired electron spins and has been extensively applied in radiation research (Lund and Shiotani 2014). In particular, EPR spectroscopy can be utilized for dosimetry purposes via quantitative analysis of EPR signal amplitudes resulting from paramagnetic centers (radicals) induced by ionizing radiation in solid materials (Regulla and Deffner 1982, Ikeya 1993, Anton 2005, ISO/ASTM 51607:2013). Usually, solid dosimeters are irradiated and then read out on a later occasion (within hours, days or even weeks) featuring a passive, off-line procedure. Since the EPR readout does not affect the radical concentration (non-destructive) in the sample, repetitive readouts are feasible.

EPR dosimeter materials should meet the following two basic requirements: i) Persistent radicals are created via irradiation and ii) the radical concentration increases monotonously with the absorbed radiation dose. Ideally, EPR dosimetry materials show a linear dependency of the radical concentration on the absorbed dose and low recombination rates, i.e. slow EPR signal fading.

One of the first and most intensively studied EPR dosimeter materials is polycrystalline L-alanine (AL) (Gordy et al 1995, Bradshaw et al 1962). AL forms very stable radicals upon irradiation (Regulla and Deffner 1982, Arber and Sharpe 1993) and the amount of radicals increases linearly with dose up to $5 \mathrm{kGy}$ (Nagy 2000). Alanine dosimetry is commonly applied in high dose (kGy) applications such as radiation processing and sterilization (Wieser and Regulla 1989, McLaughlin and Desrosiers 1995). In this dose range, elaborated dosimetry guidelines exist (ISO/ASTM 51607:2013). Further remarkable features of AL are its high water equivalence and the minor dependencies of its EPR response on beam energy (Waldeland and Malinen 2011), dose rate (Desrosiers et al 2008) and beam angle for therapeutic MV photon beams. These properties offer high potential for applications in external beam radiotherapy (EBRT) (Baffa and Kinoshita 2014).

Due to its favorable characteristics, several researchers have successfully applied alanine dosimetry in EBRT, especially for challenging dosimetry tasks such as In-vivo dosimetry (Wagner et al 2008, Schaeken et al 2010, Wagner et al 2017), small-field dosimetry (Garcia et al 2011, Vega Ramirez et al 2011), mailing dosimetry audits (Schaeken et al 2011) and intercomparisons (De Angelis et al 2005), FLASH-radiotherapy (Bourhis et al 2019) and dosimetry in magnetic fields (Billas et al 2020). Nowadays, several national institutes use alanine dosimeters as a secondary standard with a dose uncertainty $(1 \sigma)$ of less than $1 \%$ for doses above 5 Gy (Sharpe et al 1996, Anton 2005, 2006). By following these sophisticated measuring and evaluation procedures, precise dose measurements with alanine can be achieved. EPR dosimetry is thus an attractive technique with a wide range of potential applications in radiotherapy.

However, routine use of EPR dosimetry is not widespread in radiotherapy - presumably due to the reported increase in uncertainty at the 1 Gy dose level (Sharpe et al 1996, Anton 2006, Helt-Hansen et al 2009), the high costs for the investment and the space requirements of generally large-sized EPR 
spectrometers as well as the associated efforts of precise dose measurements. Against this background the question arises whether a practical and cost-efficient EPR dosimetry system utilizing a benchtop spectrometer in combination with more sensitive dosimeters may meet the accuracy requirements in radiotherapy.

In the past two decades and in order to decrease the lower limit of detection, novel EPR dosimeter materials like lithium formate monohydrate (LFM) have been proposed (Lund et al 2002, Vestad et al 2003, Lund et al 2005) and tested for dosimetry in EBRT (Vestad et al 2004, Waldeland et al 2010, Adolfsson et al 2014a, 2019) at doses around 1 Gy. Compared to AL, EPR dosimetry with LFM is more sensitive since LFM provides higher EPR signal intensities for equal dose (Lund et al 2002, Vestad et al 2003) - primarily due to its narrower EPR spectrum. In terms of the effective atomic number (Vestad et al 2003) and the energy dependence of the mass energy absorption coefficient (Lund et al 2002) as well as the mass stopping power relative to water (Vestad et al 2004, Gustafsson et a/ 2008), LFM is even more water-equivalent than AL featuring lower beam energy dependence (Waldeland and Malinen 2011) especially for $\mathrm{kV}$ beam qualities.

It is well known that the achievable level of uncertainty in EPR dosimetry is primarily depending on the dose level, the dosimeter material and its mass. In addition, the equipment used for readout as well as the measuring protocol and the spectral evaluation technique play an important role. It is therefore imperative to elucidate the actual dose uncertainty for a specific EPR dosimetry system consisting of dosimeters, EPR spectrometer, measuring protocol and spectral evaluation procedure. There is still a strong need to thoroughly improve, analyze and report uncertainty levels as performance characteristic of EPR dosimetry - especially for the novel dosimeter materials like LFM.

The purpose of the current work is to examine and to optimize the dose uncertainties of EPR dosimetry in the dose range from 1 to $70 \mathrm{~Gy}$ by using a compact and practical EPR dosimetry system - as being suitable for on-site clinical routine application in EBRT. A specific data post-processing procedure is developed for spectral evaluation. In terms of uncertainties, a direct comparison between self-pressed LFM pellets and commercial AL pellets is presented.

\section{Material and Methods}

\subsection{Dosimeter preparation and storage}

The AL pellets were received from Aérial (IIlkirch, France), whereas the LFM pellets were manufactured in-house. Lithium formate monohydrate $98 \%$ was purchased from Sigma-Aldrich (St. Louis, MO, USA). Before pressing, the powder was roughly ground with a pestle and mortar. Afterwards, the powder was portioned by weight and pressed by means of a hydraulic tablet press (Bodenseewerk Perkin-Elmer, Überlingen, Germany) without the use of additional binder material. All pellets had a diameter of $4 \mathrm{~mm}$ 
and a height of $2 \mathrm{~mm}$ (AL) or $4 \mathrm{~mm}$ (LFM). The mean pellet mass was $35.81 \pm 0.13(2 \sigma) \mathrm{mg}$ and $73.81 \pm 0.76(2 \sigma) \mathrm{mg}$ for AL and LFM, respectively. In total, 25 pellets per material were prepared and stored at constant room temperature inside an air-tight box that provided shielding from daylight as well as a constant level of $34 \pm 2 \%$ relative humidity $(\mathrm{RH})$. The relative humidity was controlled by a saturated aqueous solution of $\mathrm{MgCL}_{2}$ covering the bottom of the storage box. All pellets were stored for at least 8 weeks prior to irradiation in order to achieve an equal level of moisture content among all pellets.

\subsection{Irradiation}

For irradiation, the pellets were placed in a cylindrical air-tight polyethylene capsule with an inner and outer diameter of $4 \mathrm{~mm}$ and $6.4 \mathrm{~mm}$, respectively. Synthetic rubber plugs served as sealing. Every capsule contained two pellets - one of each type - as shown in figure 1(a).

a)

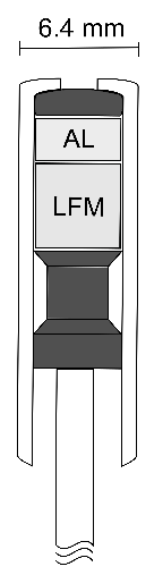

b)

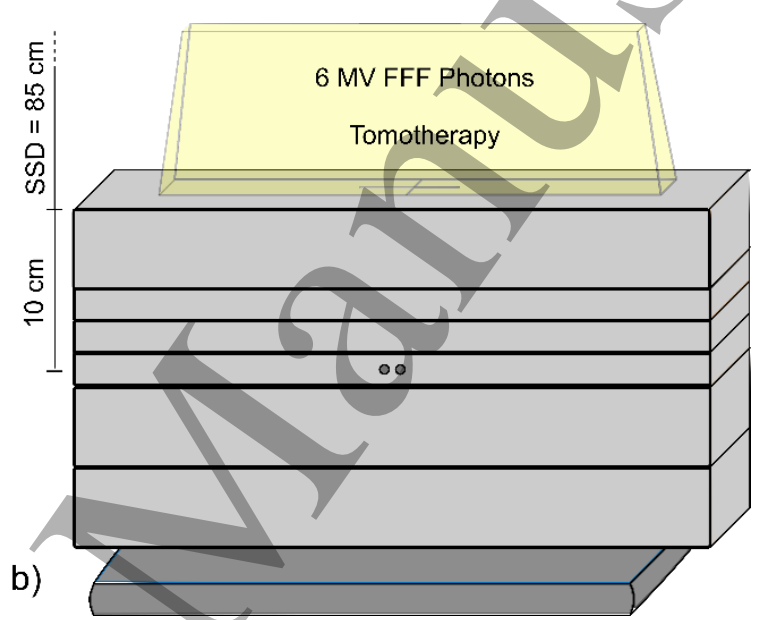

Figure 1. Cylindrical polyethylene dosimeter capsule containing one AL and one LFM pellet (a). Synthetic rubber plugs are shown as black parts. Irradiation setup utilizing a solid water slab phantom (b).

The EPR capsules were put at $10 \mathrm{~cm}$ depth inside a solid water slab phantom $(15 \mathrm{~cm} \times 55 \mathrm{~cm} \times 21 \mathrm{~cm})$ and positioned on the central axis of a clinical $6 \mathrm{MV}$ flattening filter free (FFF) photon beam of a Tomotherapy ${ }^{\circledR}$ $\mathrm{Hi}-\mathrm{Art}^{\circledR}$ (Accuray, Sunnyvale, CA, USA) treatment machine (figure 1(b)). A maximum field size of $5 \mathrm{~cm} \mathrm{x}$ $40 \mathrm{~cm}$ and $85 \mathrm{~cm}$ source surface distance (SSD) was chosen. Irradiation was applied in two subsequent fractions. In between, the capsules were rotated by $180^{\circ}$. In total, 25 capsules were irradiated and five different nominal doses were applied: 1, 5, 20, 50 or $70 \mathrm{~Gy}$. At each dose level, 5 capsules were irradiated. Absolute dose measurements were performed with a calibrated A1SL (Standard Imaging, Middleton, WI, USA) reference ionization chamber considered as the ground truth and the real absolute dose values obtained were: $0.986,4.926,19.694,49.242$ and $68.924 \mathrm{~Gy}$.

The diameter of the phantom holes at $10 \mathrm{~cm}$ depth matched the outer diameter of the ionization chamber and EPR capsules. The temperatures of the capsules during irradiation were within $25 \pm 2.5(2 \sigma)^{\circ} \mathrm{C}$. 
After irradiation, the pellets were stored at room temperature inside the storage box (see section 2.1). One additional capsule was irradiated to 273 Gy to obtain a high signal-to-noise (SNR) AL spectrum for evaluation purposes (see section 2.4).

\subsection{EPR readout}

Continuous wave (CW) EPR measurements were performed on a benchtop EPR spectrometer operating in X-band (MiniScope MS 5000, Magnettech by Freiberg Instruments GmbH, Freiberg, Germany). A magnetic field sweep width of $15 \mathrm{mT}$, a $100 \mathrm{kHz}$ modulation with amplitude of $0.8 \mathrm{mT}$ (overmodulation in order to increase the SNR) and a microwave power of $2 \mathrm{~mW}$ were applied. The microwave power setting assured operation in the linear regime, where the EPR intensity increases linearly with the square root of the microwave power (Eaton et al 2010) for both the reference and the pellet signal. Since the handling of the pellets may result in a small mass loss, every pellet mass was determined with an analytical balance (Kern ABT 120-5DM, Kern \& Sohn GmbH, Balingen-Frommern, Germany) right before EPR readout. In order to position each pellet in the center of the resonator, a dedicated sample holder system (figure 2) was used. Each pellet was inserted into a cylindrical quartz tube (hereinafter referred to as the 'sample tube') with an inner diameter of $4.1 \mathrm{~mm}$. Two different sample tubes were used-one for each dosimeter material. The pellets were supported by an PTFE insert that was affixed to the bottom of each sample tube. This insert ensured central positioning of each pellet within the sample tube and prevented possible horizontal tilts. The PTFE inserts differed in height to compensate for the different heights of the two pellet types. The sample tube containing the PTFE insert and the pellet was then inserted into the tube holder with a fixed axial orientation. The tube holder minimized lateral and horizontal variations in positioning between different readouts. During each readout, 10 subsequent scans were acquired and averaged without rotating the sample tube. Auto tuning of the resonator coupling between the scans was enabled. The time for one scan was set to $60 \mathrm{~s}$ resulting in a measurement time for a single readout of about 10 min per pellet. In order to examine the reproducibility of EPR signal amplitudes, each pellet was readout on a weekly basis within the first five weeks following irradiation.

A manganese $\left(\mathrm{ZnS}: \mathrm{Mn}^{2+}\right.$ ) reference substance (Magnettech by Freiberg Instruments $\mathrm{GmbH}$, Freiberg, Germany) was introduced into the resonator from the side of the spectrometer (figure 2) serving as an intensity reference signal during readouts. In general, the spin $\mathrm{S}=5 / 2$ system of $\mathrm{Mn}^{2+}$ provides a six-line EPR spectrum. In this work, the middle two EPR lines were recorded by the applied magnetic field sweep. 


\subsection{Spectral evaluation}

In EPR dosimetry, the EPR signal amplitudes of irradiation induced radical spectra are evaluated. For precise dose measurements, the determined amplitudes need to be corrected for several influence quantities outlined in the following. Regarding the spectrometer performance, the constancies of the modulation amplitude, microwave power, resonator quality factor $Q$ as well as sensitivity fluctuations of electronic components may affect the reproducibility of absolute EPR intensities. In order to compensate for these possible instabilities, the relative amplitude of the radical spectrum with respect to the amplitude of a well-known reference substance is typically used for precise dose measurements (Nagy et al 2000a, Anton 2005). In the current work, a $\mathrm{Mn}^{2+}$ reference substance was simultaneously measured with each pellet and served as intensity reference as well as for microwave frequency normalization of the measured spectra. As a consequence, the resulting total EPR spectrum $S$ is essentially composed of the pure radical spectrum $R$, which is the one of primary interest, and the $\mathrm{Mn}^{2+}$ spectrum $\mathrm{Mn}$. The amplitudes of the reference signal $M n$ and the radical signal $R$ were evaluated via fitting of pre-known base spectra $\left(R^{B}, M n^{B}\right)$ to the composite spectrum $S$. The final fit coefficients $a$ and $b$ reflect the amplitude modification factor of $R^{B}$ and $M n^{B}$, respectively, and result from a least-squares optimization. The spectral fitting procedure was implemented in $\operatorname{MATLAB}^{\circledR}$ (MathWorks, Nattick, MA, USA) and is exemplarily illustrated in figure 3 for AL. 


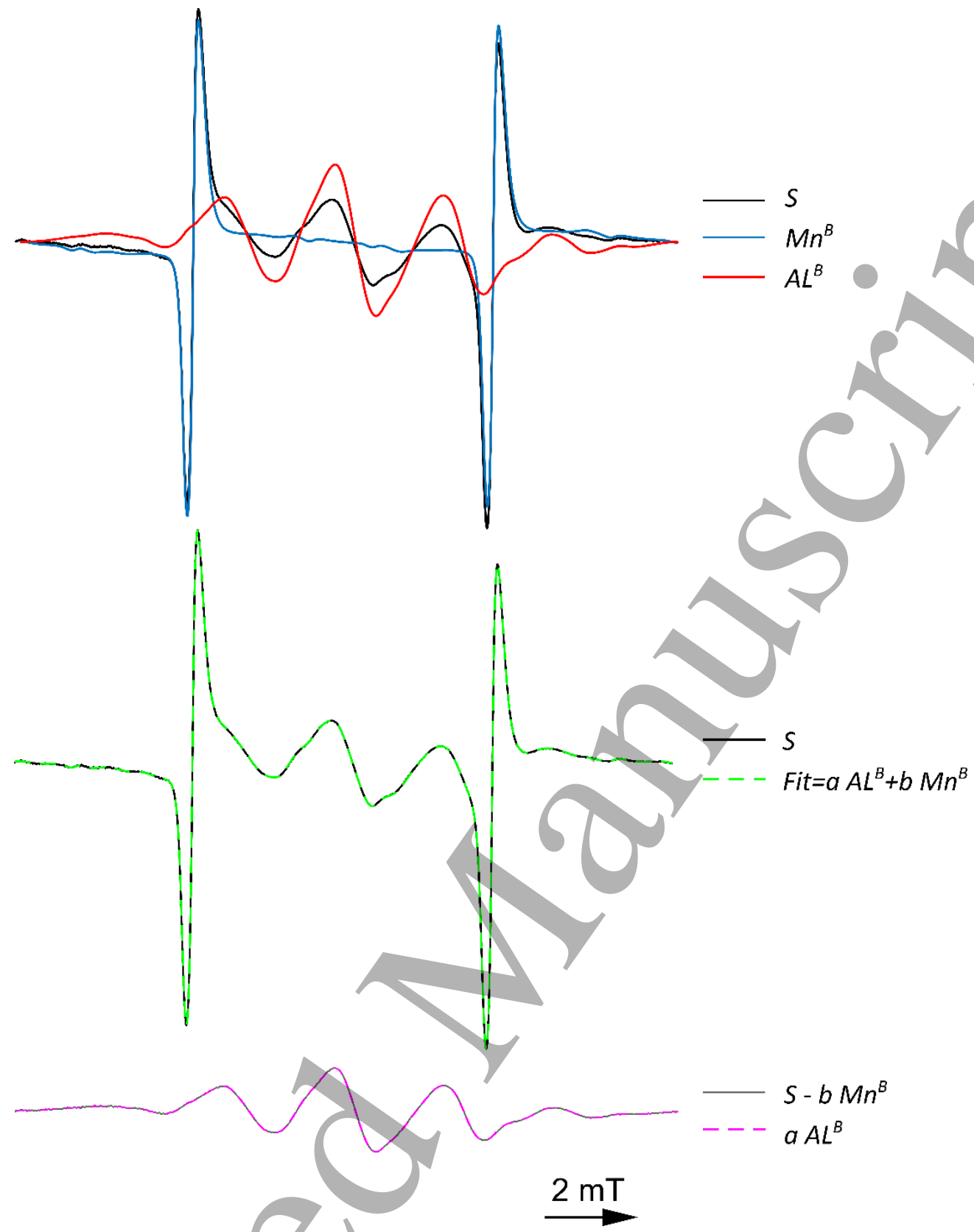

Figure 3. Example of the spectral fitting procedure for an AL pellet ( $40 \mathrm{~Gy}$ ). Measured composite spectrum $S$ (black) and base spectra: $A L^{B}$ (red) and $M n^{B}$ (blue) (top). Resulting fit (green) (middle). Extracted pure AL spectrum (purple) (bottom).

The pure radical base spectra $R^{B}$ were reconstructed from measurements of high SNR spectra (273 Gy for $A L$ and 70 Gy for LFM). These dose values yield comparable SNR for both pellet types. The manganese base spectrum $M n^{\hat{B}}$ was acquired for $1 \mathrm{~h}$ on each measurement day and for each material (AL and LFM) with a respective non-irradiated pellet inserted in the sample holder system. The $M n^{B}$ base spectrum included, in addition to the pure Mn spectrum, small EPR signal contributions from the resonator, the tube holder, the sample tube and its PTFE insert as well as the native pellet signal. 
In summary, the total EPR spectrum $S$ is primarily to be seen as a superposition of $R^{B}$ and $M n^{B}$. Additionally, the EPR spectrum is affected by a small, random, non-linear signal that varies between different readouts (Nagy et al 2002) and constitutes the residual signal (RES). All contributions are summarized in equation (1).

$$
\begin{gathered}
S=a R^{B}+b M n^{B}+R E S \\
A_{D}=\frac{a}{b} D^{B} \frac{m^{B}}{m} \frac{k_{t} k_{T}}{k_{t,}^{B} k_{T \prime}^{B}} \propto D_{i r r}
\end{gathered}
$$

The spectrometer instabilities are cancelled out when taking the ratio of the final fit coefficients $(a / b)$ for further analysis. This ratio is then multiplied by the known dose value of the $R^{B}$ spectrum $D^{B}(2)$. Further corrections concerning the sample mass $m$ with respect to $m_{B}$ (mass of the pellet used to obtain $R^{B}$ ) as well as consideration of temporal fading $k_{t}$ and the irradiation temperature $k_{T}$ need to be taken into account. By this means, the so called dose normalized amplitude $A_{b}$ (Anton 2006) is obtained that correlates more precisely with the irradiation dose $D_{\text {irr }}$ (2) than the untreated final fit coefficient $a$.

In the present work, $A_{D}$ does not include corrections for varying irradiation temperature since the effect is minor for the temperature differences encountered during irradiation (section 2.2). However, the effect is considered during the uncertainty analysis (section 2.5).

Temporal fading is a known effect in EPR dosimetry. Therefore, precise EPR dosimetry protocols correct for the associated signal loss over time. Fading rates may depend on the manufacturing process (Anton 2008) as well as on the pre- and post-irradiation storage conditions (Arber and Sharpe 1993, Dolo and Feaugas 2005). Hence, fading measurements need to be performed for each specific type of pellet and the applied storage conditions.

In case of $\mathrm{AL}$, the $\mathrm{AL}$ base spectrum $R^{B}$ was recorded only once on the first measurement day since no temporal changes in line shape were observed. The amplitude of the current $M n^{B}$ spectrum was fitted to the respective spectrum obtained on the first measurement day. The spectral fitting procedure was performed with the amplitude adjusted, current $M n^{B}$ spectrum and $R^{B}$. Amplitude fading corrections were applied according to $\frac{k_{t}}{k_{t \prime}^{B}}=f \cdot\left(t-t^{\prime}\right)$. The fading rate $f$ was assumed to be constant. It was estimated from follow-up measurements of the $70 \mathrm{~Gy}$ AL pellets within 4 months after irradiation.

For LFM, an up-to-date base spectrum $R^{B}{ }_{c}$ was derived by averaging the mass-corrected 70 Gy spectra acquired on each measurement day in order to consider possible changes in line shape over time. The fitting procedure described above was then performed with the current base spectra $\left(M n^{B}, R^{B}{ }_{c}\right)$. Hence, fading corrections were inherently included in the LFM analysis $\left(k_{t \prime}^{B}=k_{t}\right.$, since $\left.t=t^{\prime}\right)$. 
During fitting, the random residual signal RES was modeled by a $2^{\text {nd }}$ order polynomial function.

A calibration curve ( $A_{D}$ vs. $D_{\text {irr }}$ ) was obtained by a linear (3) or quadratic regression model (4). The data points were weighted by $1 / D_{\text {irr. }}\left(l_{1}, l_{0}\right)$ and $\left(q_{2}, q_{1}, q_{0}\right)$ are the resulting regression coefficients for the linear and quadratic model, respectively.

$$
\begin{aligned}
& A_{D}=l_{1} D_{i r r}+l_{0} \\
& A_{D}=q_{2} D_{i r r}^{2}+q_{1} D_{i r r}+q_{0}
\end{aligned}
$$

Calibration is needed for later estimation of unknown doses $D_{W}^{C}$ via the inverse functions (5) or (6), respectively.

$$
\begin{aligned}
& D_{W}^{C}=\frac{A_{D}}{l_{1}}-\frac{l_{0}}{l_{1}} \\
& D_{W}^{C}=\frac{1}{2 q_{2}}\left(\sqrt{q_{1}^{2}+4 q_{2}\left(A_{D}-q_{0}\right)}-q_{1}\right)
\end{aligned}
$$

\subsection{Uncertainty analysis}

In this work, a EPR dosimetry system is calibrated against the reading from a calibrated reference ionization chamber. The stated uncertainties reflect the precision of the EPR dosimetry system. For consideration of accuracy, the uncertainty of the ionization chamber calibration and the reference dosimetry procedure need to be taken into account additionally.

All measurement uncertainties in this work are determined and expressed in accordance to the Guide to the Expression of Uncertainty in Measurement published by the International Organization for Standardization (BIPM 2008). Unless otherwise stated, all uncertainties are given as standard measurement uncertainties $(1 \sigma)$.

In order to perform a Type A uncertainty analysis of the presented dosimetric procedure, a set of 25 dosimeter capsules were irradiated and evaluated five times (section 2.2). The determined relative uncertainty $\left(u_{r}\left(A_{D}^{m}\right)\right)$ for a single readout at each dose level is an estimate of the standard deviation among the $n=25 A_{D}$-values (five different pellets measured five times). The relative standard error $S E_{r}$ of this estimate is in good approximation given by $S E_{r}=u_{r}\left(A_{D}^{m}\right) / \sqrt{2(n-1)}$ (Harding et al 2014). A Type $B$ uncertainty analysis was conducted by the consideration of error propagation starting from the formulas that were used to determine $A_{D}(2)$ and the final Dose $D_{W}^{C}(5),(6)$. 


$$
\begin{gathered}
u\left(A_{D}^{C}\right)^{2}=u\left(\frac{a}{b}\right)^{2}+A_{D}{ }^{2}\left[u_{r}(m)^{2}+u_{r}(D)^{2}+\left(\frac{d k_{T}}{d T} u(T)\right)^{2}\right] \\
u\left(D_{W}^{C}\right)=\frac{1}{l_{1}} \sqrt{D_{W}^{C}{ }^{2} S E\left(l_{1}\right)^{2}+S E\left(l_{0}\right)^{2}+\left(u\left(A_{D}\right)\right)^{2}+2 D_{W}^{C} \operatorname{cov}\left(l_{1}, l_{0}\right)} \\
u\left(D_{W}^{C}\right)=\sqrt{\left(\frac{\partial D_{W}^{C}}{\partial A_{D}^{C}}\right)^{2} u\left(A_{D}^{C}\right)^{2}+\sum_{i}\left(\frac{\partial D_{W}^{C}}{\partial q_{i}}\right)^{2} S E\left(q_{i}\right)^{2}+2 \sum_{i, j} \frac{\partial D_{W}^{C}}{\partial q_{i}} \frac{\partial D_{W}^{C}}{\partial q_{j}} \operatorname{cov}\left(q_{i}, q_{j}\right)\left(1-\delta_{i j}\right)}
\end{gathered}
$$

$$
\frac{\partial D_{W}^{C}}{\partial q_{2}}=\frac{1}{q_{2}}\left(\frac{A_{D}-q_{0}}{2 q_{2} D_{W}^{C}+q_{1}}-D_{W}^{C}\right)
$$

The following estimations concerning the uncertainty budget were made: the standard uncertainty $(1 \sigma)$ of the pellet mass is $u(m)=0.05 \mathrm{mg}\left(u_{r}(m)=0.13 \%(A L), u_{r}(m)=0.06 \%(L F M)\right)$, the reproducibility of irradiation is $u_{r}(D)=0.2 \%$ and the uncertainty contributions from varying irradiation temperatures are $\frac{d k_{T}}{d T} u(T)=0.20 \%(A L), \frac{d k_{T}}{d T} u(T)=0.19 \%(L F M)$ with $u(T)=1.25 \mathrm{~K}$ and $\frac{d k_{T}}{d T}=$ $0.161 \% / K$ for AL or $\frac{d k_{T}}{d T}=0.154 \% / K$ for LFM (Waldeland et al 2011). The uncertainty of the analyzed EPR amplitudes $u\left(\frac{a}{b}\right)$ was determined by a least-squares fit of $u_{r}\left(A_{D}^{C}\right)$ to the measured uncertainties $u_{r}\left(A_{D}^{m}\right)$ (section 3.3) for each material. Overall, the uncertainty of the dose normalized amplitude $u\left(A_{D}^{C}\right)$ is given by (7).

In a second step, when the irradiation dose is unknown, the fit coefficients obtained via calibration can be applied to calculate the dose $D_{W}^{C}$ from measured $A_{D}$-values via (5) or (6), respectively. In order to calculate the resulting standard uncertainty $u\left(D_{W}^{C}\right)$, the standard errors $S E$ of the regression coefficients and their covariances cov need to be considered as well. Equation (8) shows the formula in case of a linear regression model. The corresponding formula for the quadratic model is likewise obtained through error propagation (9) - (12). 


\section{Results}

Post-processed (section 2.4) EPR spectra of AL and LFM pellets irradiated to $70 \mathrm{~Gy}$ are depicted in figure 4 for comparison. The amplitudes were corrected for the pellet mass in order to show the effect of the dosimeter material on the EPR signal amplitude and the spectral width.

Irradiation induced radicals in LFM reveal a narrower EPR spectrum than AL radicals. Moreover, the LFM peak-to-peak amplitudes are about twice as high when comparing LFM and AL pellets of equal dose and mass.

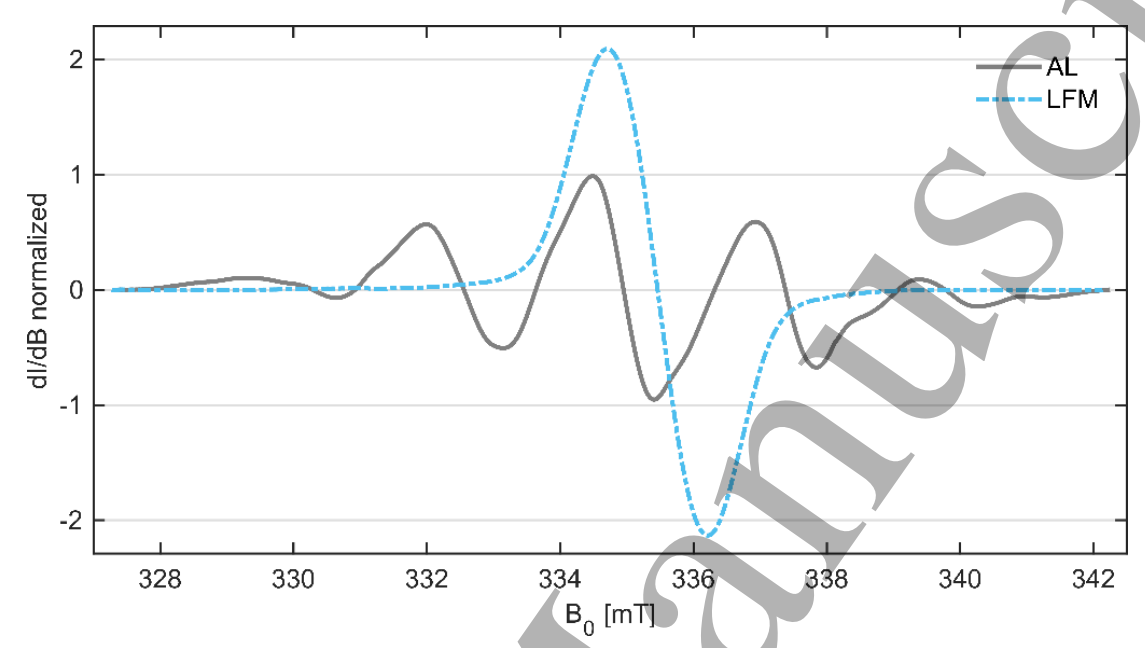

Figure 4. Comparison of mass corrected AL and LFM EPR spectra obtained from pellets irradiated to $70 \mathrm{~Gy}$. The intensities are normalized to the maximum of the AL EPR spectrum.

\subsection{Temporal fading}

Long-term measurements were conducted for the 70 Gy pellets in order to examine the EPR signal loss after irradiation as a function of time. In case of AL, low fading rates extrapolated to about $f=3 \% / a$ were obtained (data not shown) and no changes in EPR line shape were observed. For LFM, however, notable temporal changes in line shape were detected. Figure 5 shows the base spectra reconstructed from the 70 Gy spectra recorded on day 4, day 32 and day 67 after irradiation. The EPR spectral width of irradiated LFM pellets decrease slightly during the first five weeks following irradiation (day 4 vs. day 32) gradually approaching a steady line shape (day 32 vs. day 67). In turn, the peak-to-peak signal amplitudes increase by about $3 \%$ with respect to the initial spectrum (day 4) within the first five weeks. These temporal changes in line shape and peak-to-peak amplitude were considered during the evaluation of LFM spectra by using an updated LFM base spectrum on each measurement day (section 2.4). 


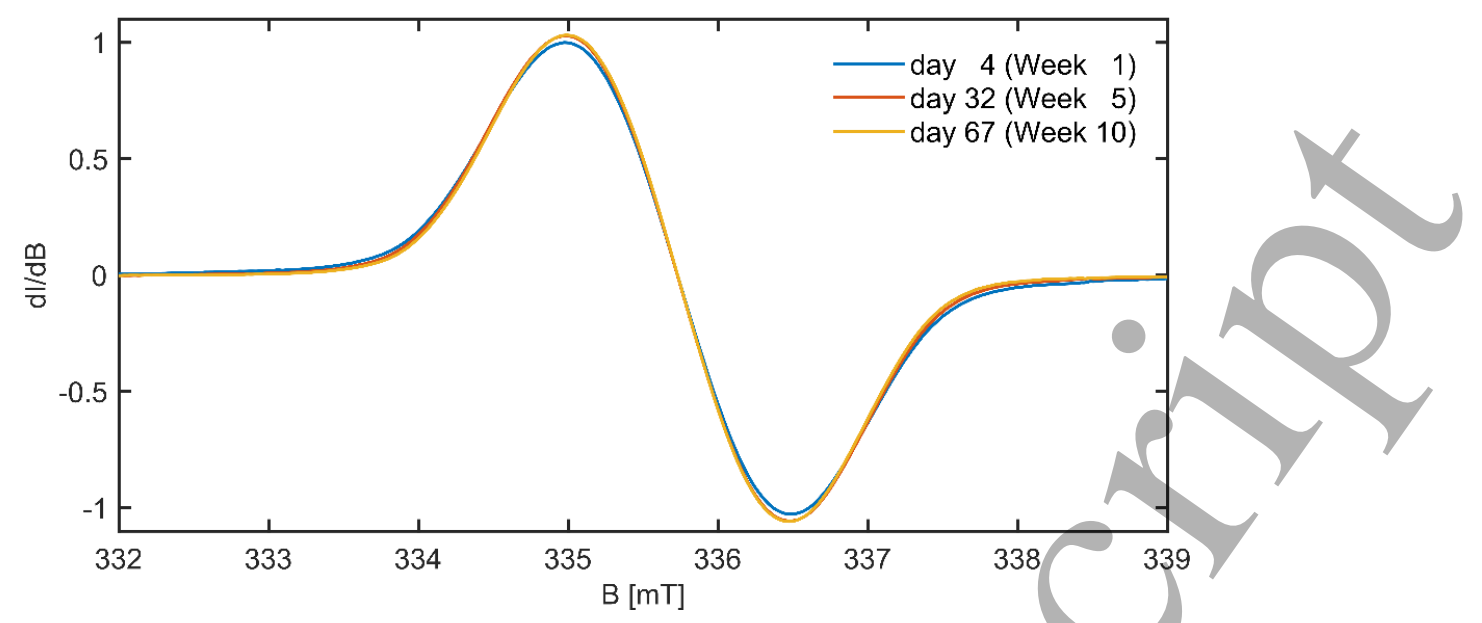

Figure 5. Comparison of spectral line shapes and amplitudes of LFM base spectra (70 Gy) recorded at different time points after irradiation. The amplitudes are normalized to the maximum signal intensity of the initial spectrum (day 4).

\subsection{Calibration}

In total, 125 readouts were performed for each dosimeter type, i,e. 25 readouts per dose level. The resulting dose values $\left(A_{D}\right)$ obtained by the elaborated EPR dosimetry procedure are shown in the top graphs of figure 6 for AL (left) and LFM (right). Both dosimeter materials show a nearly linear dependency between the measured dose values and the irradiation dose $\left(D_{i r r}\right)$. The displayed line indicates a true linear dependency $\left(A_{D}=D_{i r r}\right)$.

Relative variability of the $A_{D}$ values around this line is depicted in the middle graphs of figure 6 highlighting the increase in relative variance at lower doses. Linear $(I m)$ and quadratic models ( $q m$ ) obtained via regression analysis are shown as blue and purple curves, respectively. The mean relative residuals as well as the $95 \%$ confidence bounds of the respective model are given in the bottom graphs of figure 6. The error bars indicate the standard errors of the mean relative residuals (SEM). In case of AL, the linear model predicts the mean $A_{D}$-value adequately, as the mean relative residuals $( \pm S E M)$ are located within the $95 \%$ confidence bounds for all dose levels. This situation is different for the LFM data. Here, the mean relative residuals of the linear model lie outside of the confidence bounds. As a consequence, a quadratic model was chosen which predicts the mean $A_{D}$-values more accurately, i.e. for the quadratic model, the mean relative residuals $( \pm S E M)$ are located within the $95 \%$ confidence bounds. 

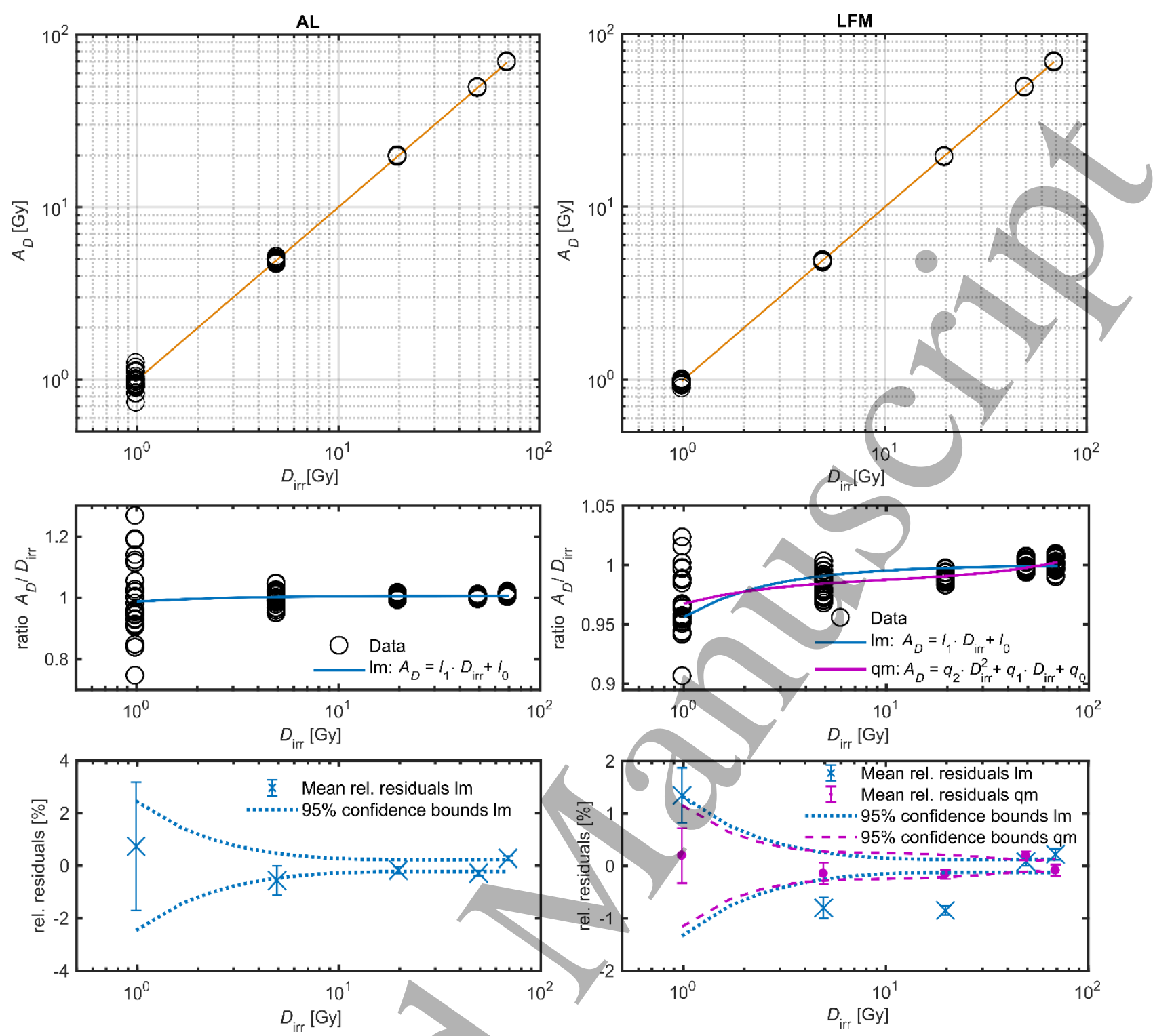

Figure 6. Calibration results for AL (left) and LFM (right). Determined dose normalized amplitudes $A_{D}$ vs. irradiation dose $D_{\text {irr }}$ (black circles) and straight line through the origin with slope 1 (brown) (top row). Ratios $A_{D} / D_{\text {irr }}$ vs. $D_{\text {irr }}$ (black circles) and fitted linear (blue) and quadratic (purple) models (middle row). Relative mean deviations (residuals) of the data points from the respective fit and $95 \%$ confidence bounds of the model (bottom row). The error bars indicate the standard errors of the mean residuals. 
Detailed results of the regression analysis are listed in table 1. The standard errors (SE) of the fit coefficients and the covariances reflect the uncertainty of calibration. Next, these uncertainties contribution are taken into account for a complete uncertainty analysis of the dosimetric procedure.

\begin{tabular}{|c|c|c|c|c|c|c|c|}
\hline & \multirow{2}{*}{\multicolumn{2}{|c|}{$\frac{\text { AL }}{\text { linear }}$}} & \multicolumn{5}{|c|}{ LFM } \\
\hline \multirow{3}{*}{$\begin{array}{c}\text { Model } \\
\text { Regression } \\
\text { coefficients }\end{array}$} & & & \multicolumn{2}{|c|}{ linear } & \multicolumn{3}{|c|}{ Quadratic } \\
\hline & $l_{1}$ & $l_{0}$ & $l_{1}$ & $l_{0}$ & $q_{2}$ & $q_{1}$ & $q_{0}$ \\
\hline & 1.006 & -0.019 & 1.000 & -0.043 & $2.2 \mathrm{e}-4$ & 0.987 & -0.020 \\
\hline \multirow{2}{*}{$\begin{array}{c}\text { Standard } \\
\text { error }\end{array}$} & $S E\left(l_{1}\right)$ & $S E\left(l_{0}\right)$ & $S E\left(l_{1}\right)$ & $S E\left(l_{0}\right)$ & $S E\left(q_{2}\right)$ & $S E\left(q_{1}\right)$ & $S E\left(q_{0}\right)$ \\
\hline & 0.001 & 0.013 & 0.001 & 0.007 & 3 e-5 & 0.002 & 0.007 \\
\hline \multirow{2}{*}{ Covariance } & \multicolumn{2}{|c|}{$\operatorname{cov}\left(l_{1}, l_{0}\right)$} & \multicolumn{2}{|c|}{$\operatorname{cov}\left(l_{1}, l_{0}\right)$} & $\operatorname{cov}\left(q_{2}, q_{1}\right)$ & $\operatorname{cov}\left(q_{1}, q_{0}\right)$ & $\operatorname{cov}\left(q_{2}, q_{0}\right)$ \\
\hline & \multicolumn{2}{|c|}{-5.54 e- 6} & \multicolumn{2}{|c|}{-1.63 e- 6} & -0.05 e- 6 & $-6.54 e-6$ & 0,10 e-6 \\
\hline
\end{tabular}

Table 1. Results of the linear and quadratic prediction models resulting from regression analysis of AL and LFM data.

\subsection{Uncertainty analysis}

Relative standard deviations $u_{r}\left(A_{D}^{m}\right)$ were estimated from the 25 measurements at each dose level and for each dosimeter type. The results are shown in figure 7 for AL (a) and LFM (b) as black circles. As can already be seen in figure 6 , the scattering of the $A_{D}$ values increases with decreasing irradiation dose for both dosimeter types. In figure 7, a rapid increase in the standard deviation is observed at doses below 5 Gy. At the 1 and 5 Gy dose level the relative uncertainty $u_{r}\left(A_{D}^{m}\right)$ is considerably smaller for LFM (by a factor of about 4) compared to AL. At higher dose levels (>20 Gy) a relative uncertainty $u_{r}\left(A_{D}^{m}\right)<0.7 \%$ was observed for both materials.

Equation (7) was applied to estimate the uncertainty of the $A_{D}$ values $u_{r}\left(A_{D}^{c}\right)$ from the uncertainty budget for arbitrary dose values within the investigated dose range. In case of $A L$, a dose independent contribution of $0.13 \mathrm{~Gy}$ was assumed reflecting the uncertainty of the analyzed EPR signal amplitudes $u\left(\frac{a}{b}\right)$, i.e. the sensitivity of the dosimetry system. For LFM, $u\left(\frac{a}{b}\right)$ was set to 0.03 Gy (about a factor of 4 smaller). The $u_{r}\left(A_{D}^{c}\right)$ values thus obtained (cyan dots in figure 7) are within the error range of the experimental values. The constant contribution to uncertainty $u\left(\frac{a}{b}\right)$ is the dominant uncertainty contribution at low doses ( $<20 \mathrm{~Gy}$ ). It is attributed to a random residual signal. This conclusion is supported by the fact, that the uncertainty can be reduced considerably by averaging multiple $A_{D}$ values obtained at different days. The red points in figure 7 show the resulting uncertainties when five $A_{D}$ values - obtained for the same pellet on different days - are averaged.

Since the comparison of the uncertainties associated with the AL and LFM pellets is biased by the fact that the LFM pellets contain approximately twice as much active material than the AL pellets, relative uncertainties $u_{r}\left(A_{D}^{c}\right)$ for AL pellets having the same mass as the LFM pellets were derived from equation (7) by setting $u\left(\frac{a}{b}\right)$ to $0.06 \mathrm{~Gy}$ and $u_{r}(m)$ to $0.06 \%$. The results are represented by the grey line in figure $7($ a). 
The final relative uncertainty $u_{r}\left(D_{w}^{c}\right)$ of the EPR dosimetry system is represented by the blue curves in figure 7 taking into account additionally the uncertainty of the fit coefficients obtained from the regression analysis. However, this contribution turns out to be marginal $\left(u_{r}\left(D_{w}^{c}\right)-u_{r}\left(A_{D}^{c}\right)<0.1 \%\right)$ for both materials.

In summary, the relative dose uncertainties for a single EPR readout at doses $\geq 5$ Gy are below $2.8 \%(\mathrm{AL})$ and $1.1 \%$ (LFM) but increase to $12.3 \%(\mathrm{AL})$ and $2.6 \%$ (LFM) at $1 \mathrm{~Gy}$. By averaging five independent readouts, the uncertainties at 1 Gy decrease to $2.6 \%(A L)$ and $0.8 \%(L F M)$. When $A L$ pellets with the same mass as the LFM pellets are used, the rel. uncertainties for $A L$ are expected to decrease to $6.2 \%$ at $1 \mathrm{~Gy}$ and stay below $1.3 \%$ at doses above $5 \mathrm{~Gy}$.
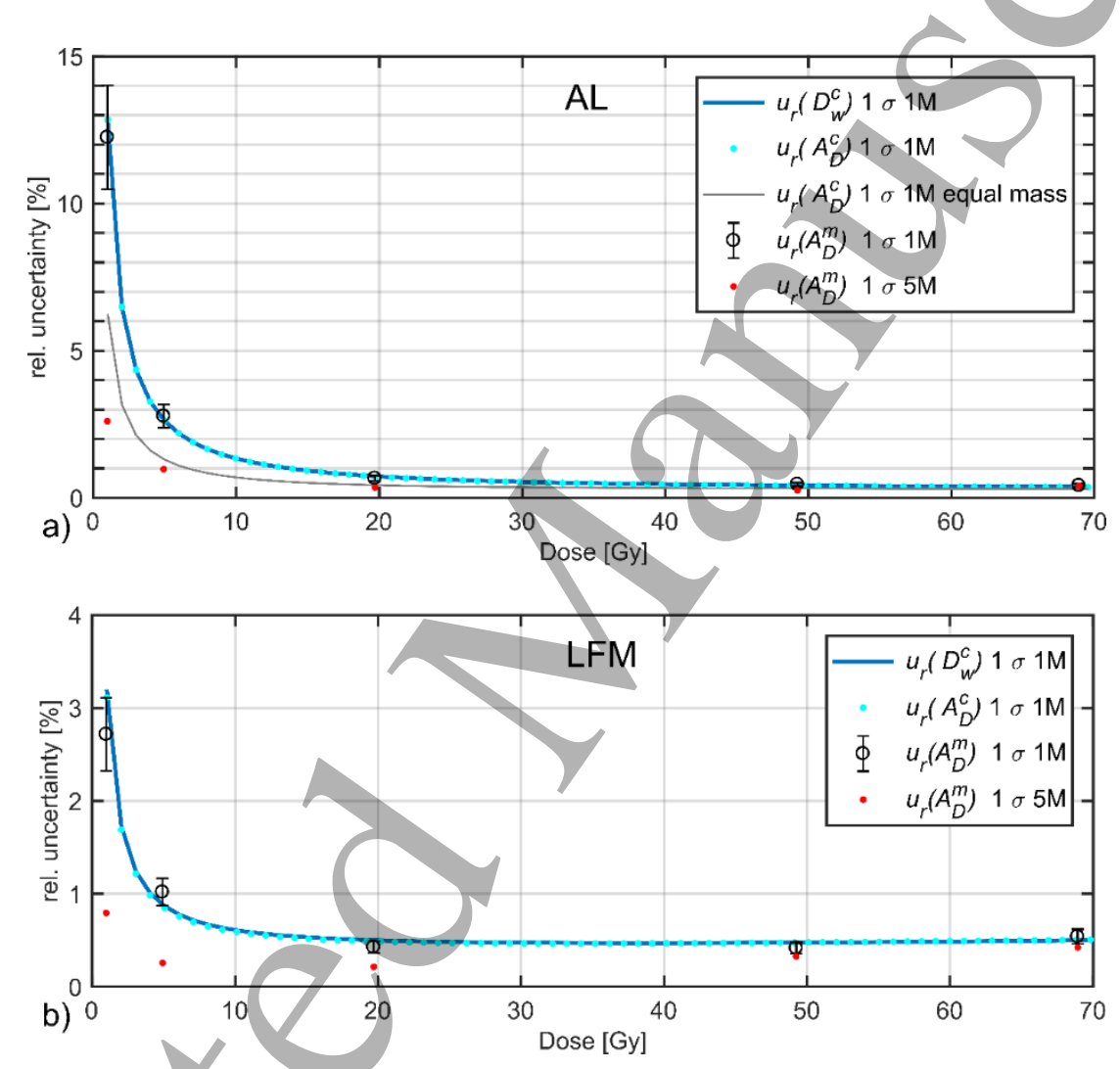

Figure 7. Analysis of relative uncertainty for $\mathrm{AL}(\mathrm{a})$ and LFM (b). Measured relative uncertainties (black circles), estimated relative uncertainties from the uncertainty budget (cyan dots) and final uncertainty including the uncertainties from the regression analysis (blue lines). The red dots represent the measured uncertainty when five dose values $\left(A_{D}\right)$ from different readouts are averaged. Expected rel. uncertainty for AL pellets with same mass as the LFM pellets (grey line in (a)). 


\section{Discussion}

The current work presents a practicable EPR dosimetry system being suitable for on-site measurements in clinical routine. A thorough investigation on the dosimetric performance of an elaborated dosimetry procedure was conducted for AL and LFM dosimeters in the dose range from 1 to $70 \mathrm{~Gy}$ using a benchtop EPR spectrometer.

For doses above $20 \mathrm{~Gy}$, similar levels of precision were found for LFM $\left(u_{r}\left(D_{w}^{c}\right)<0.5 \%\right)$ and $\mathrm{AL}\left(u_{r}\left(D_{w}^{c}\right)<\right.$ $0.7 \%)$. At lower doses (<20 Gy), however, a significantly higher precision was observed for the self-pressed LFM pellets compared to the commercial AL pellets (figure 7). Relative measurement uncertainties are known to increase with decreasing dose (i.e. EPR signal amplitudes), especially in the therapy dose range (Anton 2006). The higher precision for LFM pellets is attributed to the increased EPR signal intensities that can be explained by the following facts: i) The mass of the actual LFM dosimeters was about twice the AL pellet mass resulting in higher EPR intensities due to the approximately doubled amount of radical species. ii) The radical yield ( $G$-value) is slightly larger $\left(G_{\mathrm{LFM}} / G_{A \mathrm{~L}} \approx 1.1\right)$. iii) The width of the EPR spectrum is considerably smaller for LFM (figure 4). In general, a smaller spectrum results in higher EPR intensities, given the same amount of radicals in the sample. Here, the peak-to-peak intensities of LFM spectra are about two times higher compared to AL (figure 4) which is in good agreement with earlier reports (Komaguchi et al 2007). In total, these facts result approximately in a four times higher peak-to-peak amplitude for our LFM pellets when equal dose spectra are compared.

Experimentally obtained relative uncertainties were explained by a Type B uncertainty analysis (figure 7) throughout the investigated dose range. The analysis showed that a constant, dose independent term $u\left(\frac{a}{b}\right)(7)$ is responsible for a sharp increase in relative uncertainty at low doses (<20 Gy). $u\left(\frac{a}{b}\right)$ reflects the uncertainty of the EPR signal amplitudes resulting from the spectral fitting procedure. It is primarily attributed to small variations in the empty tube EPR spectrum arising from positional variations of the sample tube. We found that $u\left(\frac{a}{b}\right)$ expressed in terms of dose is 0.03 Gy for LFM and 0.13 Gy for AL, i.e. by a factor of about 4 smaller for LFM. The latter finding can be explained by the four times higher peak-topeak amplitude of the LFM pellets. If AL pellets with the same mass as the LFM pellets are used, $u\left(\frac{a}{b}\right)$ is expected to decrease to about $0.06 \mathrm{~Gy}$ resulting in reduced rel. uncertainties for AL (figure 7 (a)).

Our analysis suggests that the main uncertainty contribution at low doses originates from the residual signal with an average EPR signal amplitude that is independent of the dosimeter material. Moreover, averaging over several dose values obtained from the same pellet on different days resulted in a considerable decrease in relative uncertainty at low doses (figure 7). In previous works, the main contribution to $u\left(\frac{a}{b}\right)$ was attributed to inter-pellet variations in the native pellet signals (Wieser et al 1993, Haskell et al 1998, Anton 2005 and others). Anton, for example, determined $u\left(\frac{a}{b}\right)$ to be in the order of 20 mGy for AL due to inter-pellet signal variations (Anton 2005). Based on the findings of our experiments, it 
is concluded that for our dosimetry system the main contributions to the residual signal is not based on inter-pellet variations in the native pellet signals, but is rather due to geometric variations of the empty tube (as mentioned above), low frequency noise of the utilized EPR spectrometer, as well as signal anisotropy. Similar findings were reported earlier by Nagy (Nagy et al 2002). We therefore observed that the dose uncertainty could be considerably reduced when several independent readouts of the same pellet were averaged, but at the expense of practicability. Another approach of improving measuring reproducibility is averaging over several measurements performed at different angles by rotating the sample tube in between measurements. This technique is used by most of the calibration laboratories that make high accuracy measurements with alanine dosimeters (e.g. Anton 2005). Similar to the procedure presented in this study, it is expected that rel. measurement uncertainties can be further decreased by averaging the results of spectra acquired under different tube angles, since positional variations of the sample tube constituting the random residual signal will likewise be averaged out.

In summary, the dose dependency of the relative uncertainty (figure 7) is explained as follows: The relative amplitude of the residual signal with respect to the radical signal increases for low doses $(\propto 1 / D)$ thus leading to an increase in relative uncertainty. For higher doses, the contribution of the residual signal to relative uncertainty is minor. Here, the other contributions in equation (7) are dominant giving rise to a constant level of relative uncertainty.

In case of LFM, the measured uncertainties of the dosimetry system presented in this work are only slightly higher than the uncertainties stated by national metrology institutes (NMIs) for AL dosimeters: At the 5 Gy dose level, relative uncertainties achieved by alanine dosimetry at NMIs are $0.6 \%$ (Anton 2006) and $1 \%$ (Sharpe et al 1996). At this dose level, we observed a relative uncertainty of $2.8 \%$ for AL and $1.1 \%$ for LFM. The higher level of precision at the German NMI (Physikalisch-Technische Bundesanstalt (PTB)) is obtained by the use of a large-size spectrometer in combination with a high-sensitivity resonator, $\mathrm{AL}$ pellets of higher mass $(60 \mathrm{mg}$ ), a goniometer for rotating the sample tube and daily calibration of the dosimetry system (Anton 2006) resulting in a time-consuming procedure ( $8 \mathrm{~h}$ ). The procedure proposed in this work is tailored to efficiency and expediency aiming at routine application in clinical routine. It requires only moderate effort through one-time calibration, considerably less space (benchtop spectrometer) and features a convenient and short measuring protocol (readout time $10 \mathrm{~min}$ per pellet without rotating the pellet between scans). The manganese base spectrum $\mathrm{Mn}^{B}$ needs to be acquired once on each measurement day.

When necessary, dose uncertainties can be further improved by averaging independent readouts (e.g. $u_{r}\left(D_{w}^{c}\right)$ for LFM is below $1 \%$ throughout the investigated dose range when five readouts are averaged, figure 7). The effect of averaging multiple readouts of LFM dosimeters was investigated previously (Adolfsson et al 2014b) and a significant reduction in relative uncertainty was thereby achieved for low signal amplitudes. Relative uncertainties may be even further decreased by using high purity LFM (only 
98\% was used in this work), since the impurity content can affect native pellet signals and inter-pellet variability of the EPR line shape.

LFM dosimeters are thus a promising alternative to $\mathrm{AL}$ at the therapy dose level, especially when using compact spectrometers with likely lower sensitivity. In previous works, successful application of LFM dosimeters at the radiotherapy dose level was reported (Adolfsson et al 2014a, 2014b, 2019, Gustafsson et al 2008, Vestad et al 2004, Waldeland et al 2010). However, a thorough investigation of measurement uncertainties in a wide dose range, as given by Anton (Anton 2006) for AL, has not yet been reported for LFM.

Post-irradiation signal fading was examined for each material. The signal loss of $3 \% /$ a that was observed for the AL pellets is in good agreement with previously reported findings for Aérial pellets (Secerov et al 2016). For LFM, changes in line shape and intensity were observed (figure 5), especially within the first five weeks following irradiation. This is partially in contradiction with the findings of previous works. Vestad et al reported no changes in intensity and line shape within one week after irradiation (Vestad et al 2003). In their work, LFM pellets were manufactured without additional binder (as in the present work) and then irradiated to $10 \mathrm{~Gy}$ by $220 \mathrm{kV}$ x-rays. Komaguchi et al observed signal fading in LFM pellets of about $4-10 \%$ within two months after irradiation (Komaguchi et al 2007). Their pellets were pressed without binder and were irradiated to $1 \mathrm{kGy}$ by ${ }^{60} \mathrm{Co} \mathrm{y}$-rays. The radical decay was found to be dependent on the ambient relative humidity. Further, it was assumed that the signal loss is due to the reaction of the induced $\mathrm{CO}_{2}{ }^{-}$radical with stoichiometrically excessive water. Costa et al observed a significant signal decrease of about $6.6 \%$ within the 31 days after irradiation with protons for LFM dosimeters containing $10 \%$ paraffin as binder (Costa et al 2019). Also Adolfsson et al added 10\% paraffin binder during manufacturing but did not observe any significant signal loss during the first month following irradiation to $23 \mathrm{~Gy}$ by a $6 \mathrm{MV}$ photon beam (Adolfsson et al 2012). In their study, the pellets were stored in a desiccator at $33 \% \mathrm{RH}$. More recently, two additional minor radical species in irradiated LFM crystals were identified (Krivokapić et al 2015). It was shown that the EPR signal loss at ambient conditions is dominated by the decay of the major $\left(\mathrm{CO}_{2}^{-}\right)$radical leading to temporal changes in EPR line shape. Our findings are supported and may be explained by the latter investigation. In summary, the influence of binder material and environmental parameters on post-irradiation signal fading in LFM is not yet fully understood and needs to be further investigated. Also the purity grade of LFM may affect signal fading. This was beyond the scope of the current study. For precise dose evaluation, however, the temporal changes in EPR line shape and intensity need to be known for a specific batch of LFM dosimeters and need to be taken into account when applying the presented spectral fitting procedure.

Furthermore, we noted a slight non-linear dose response for LFM during calibration (figure 6). The regression analysis included all EPR readouts that were performed within five weeks after irradiation. Therefore, it cannot be ruled out that the observed non-linearity is caused by a dose dependent fading 
effect. Thus, our results support the assumption made by Komaguchi et al, who supposed that fading is due to stoichiometrically excessive water in the crystals and that fading may therefore become more prominent at low doses (Komaguchi et al 2007).

The dosimetry procedure applied in the current work accounts for fading and the observed non-linearity (LFM), thus being able to exploit the full potential of precise low dose ( $<20 \mathrm{~Gy}$ ) EPR dosimetry by using LFM dosimeters in a routine clinical environment. The uncertainty analysis showed that precise dose measurements down to $1 \mathrm{~Gy}$ are feasible, especially when using LFM dosimeters and multiple readouts. When calibrated properly, also high levels of accuracy $(<3 \%)$, as they are required in clinical dosimetry, are achievable. The presented dosimetry system is considered suitable for on-site applications in radiotherapy departments due the small-sized equipment and the restricted efforts (single calibration, measurement time of 10 min per pellet). However, as for any other dosimetry technique, proper staff training (dosimeter handling, dose evaluation, adherence to strict measuring protocols) is also a prerequisite for precise EPR dose measurements.

For less-controlled circumstances and environments (e.g. in routine clinical practice or especially when applied in-vivo), however, the overall uncertainty of the presented dosimetry system may increase, since several influencing factors may have stronger impacts or need to be taken into account additionally. For example, our uncertainty analysis was based on an irradiation temperature of $25 \pm 2.5(2 \sigma){ }^{\circ} \mathrm{C}$. If dosimeters are applied in-vivo where irradiation temperatures of up to $37^{\circ} \mathrm{C}$ are expected and if no further temperature correction is applied, the presented procedure will lead to a systematic dose error of up to $2.0 \%$ for both pellet types. In these situations, it is therefore recommended to estimate the mean and the standard deviation of the irradiation temperatures in order to perform a temperature correction according to (2) when determining $A_{D}$ and finally to consider higher values for $u(T)$ when calculating $u\left(A_{D}\right)$ via (7). For $30 \pm 7(2 \sigma)^{\circ} \mathrm{C}$ this will result in an increase of the relative dose uncertainty $(1 \sigma)$ by about $0.25 \%$ with respect to the values depicted in figure 7 .

Regarding signal fading of LFM dosimeters, the temporal evolution of the EPR line shape following irradiation was analyzed on a weekly basis after averaging the spectra of five high dose (70 Gy) samples. Thus, a set of base spectra $\left\{R^{B}\right\}$ were obtained (one on each day of measurement). The respective base spectrum was then used during spectral evaluation. When applying the presented dosimetry system in routine clinical practice, it is not necessary to irradiate additional samples in order to acquire the $R_{c}^{B}$ on each day of measurement. We suggest to ether use the nearest available base functions out of the pre-known set $\left\{R_{c}^{B}\right\}$ that was acquired during calibration or to use interpolated spectra, when samples are evaluated at different time points after irradiation. If the set $\left\{R_{c}^{B}\right\}$ is used, the additional contribution to the relative uncertainty is estimated from the differences in the peak-to-peak amplitudes to be approximately $0.3 \%$. Applying an interpolated base spectrum may considerably reduce this additional uncertainty. 
The current work presents fundamental results in order to bring EPR dosimetry from the calibration laboratories to hospital environments and will stimulate further research on applications of EPR dosimetry within clinical quality assurance programs (e.g. small field dosimetry, in-vivo dosimetry) in the future.

\section{Conclusion}

Precise dose measurements at the therapeutic dose level (1-70 Gy) are feasible, on a EPR benchtop spectrometer by implementing a practicable EPR dosimetry procedure. In terms of uncertainty at low doses ( $<20 \mathrm{~Gy}$ ), the self-made LFM pellets are superior to the AL pellets received from the manufacturer owing to their narrower EPR spectrum and approximately doubled mass resulting in higher EPR signal intensities. However, careful calibration and the knowledge of fading characteristics are prerequisites when analyzing LFM signals. By following the elaborated dosimetry procedure, relative dose uncertainties of less than $3 \%$ at $1 \mathrm{~Gy}$ can be achieved for LFM based upon a single readout (10 $\mathrm{min}$ ) and daily recording of the manganese reference signal for $1 \mathrm{~h}$. The uncertainties can be further decreased by averaging multiple dose values from independent readouts. In principle, the presented dosimetry system is considered suitable for future on-site applications in EBRT. However, overall uncertainties may increase due to less-controlled circumstances in clinical routine. For example, irradiation temperatures may be more variable when dosimeters are applied in-vivo.

\section{Acknowledgements}

All authors disclose any potential conflict of interest. 


\section{Reference}

Anton M 2005 Development of a secondary standard for the absorbed dose to water based on the alanine EPR dosimetry system Applied Radiation and Isotopes 62 779-95

Anton M 2006 Uncertainties in alanine/ESR dosimetry at the Physikalisch-Technische Bundesanstalt Physics in Medicine and Biology 51 5419-40

Anton M 2008 Postirradiation effects in alanine dosimeter probes of two different suppliers Physics in Medicine and Biology 531241

Adolfsson E, Karlsson M, Carlsson G A, Tedgren Å C, Lund E, Sara Olsson and Gustafsson H 2012 Investigation of signal fading in lithium formate EPR dosimeters using a new sensitive method Physics in Medicine and Biology 572209

Adolfsson E, Gustafsson H, Lund E, Alm Carlsson G, Olsson S and Carlsson Tedgren $\AA$ 2014a A system for remote dosimetry audit of 3D-CRT, IMRT and VMAT based on lithium formate dosimetry Radiotherapy and Oncology $113279-82$

Adolfsson E, Carlsson Tedgren Å, Alm Carlsson G, Gustafsson H and Lund E 2014b Optimisation of an EPR dosimetry system for robust and high precision dosimetry Radiation Measurements 70 21-8

Adolfsson E, Wesolowska P, Izewska J, Lund E and Tedgren A C 2019 END-TO-END AUDIT: COMPARISON OF TLD AND LITHIUM FORMATE EPR DOSIMETRY Radiat Prot Dosimetry Online: https://academic.oup.com/rpd/advance-article/doi/10,1093/rpd/ncy289/5424002

Arber J M and Sharpe P H G 1993 Fading characteristics of irradiated alanine pellets: The importance of pre-irradiation conditioning Applied Radiation and Isotopes 44 19-22

Baffa O and Kinoshita A 2014 Clinical applications of alanine/electron spin resonance dosimetry Radiat Environ Biophys 53 233-40

BIPM, IEC, IFCC, ILAC, ISO, IUPAC, IUPAP and OIML JCGM 1002008 Evaluation of Measurement Data-Guide to the Expression of Uncertainty in Measurement (Geneva: International Organization for Standardization)

Billas I, Bouchard H, Oelfke U, Shipley D R, Gouldstone C A and Duane S 2020 Alanine dosimetry in strong magnetic fields: use as a transfer standard in MRI-guided radiotherapy Phys. Med. Biol. Accepted manuscript Online: http://iopscience.iop.org/10.1088/1361-6560/ab8148

Bourhis J, Sozzi W J, Jorge P G, Gaide O, Bailat C, Duclos F, Patin D, Ozsahin M, Bochud F, Germond J-F, Moeckli R and Vozenin M-C 2019 Treatment of a first patient with FLASH-radiotherapy Radiotherapy and Oncology 139 18-22

Bradshaw W W, Cadena D G, Crawford G W, Spetzler H A W 1962 The Use of Alanine as a Solid Dosimeter. Radiation Research, 17 11-21

Costa T, Adolfsson E, Fager M and Lund E 2019 CHARACTERIZATION OF A LITHIUM FORMATE EPRDOSIMETRY SYSTEM FOR PROTON RADIATION THERAPY Radiat Prot Dosimetry 186 83-7 
S Höfel et al. Manuscript submitted to Physics in Medicine and Biology

De Angelis C, De Coste V, Fattibene P, Onori S and Petetti E 2005 Use of alanine for dosimetry intercomparisons among Italian radiotherapy centers Applied Radiation and Isotopes 62 261-5

Desrosiers M F, Puhl J M, and Cooper S L 2008 An Absorbed-Dose/Dose-Rate Dependence for the AlanineEPR Dosimetry System and Its Implications in High-Dose lonizing Radiation Metrology Journal of Research of the National Institute of Standards and Technology 113

Dolo J M and Feaugas V 2005 Analysis of parameters that influence the amplitude of the ESR/alanine signal after irradiation Applied Radiation and Isotopes 62 273-9

Eaton G R, Eaton S S, Barr D P and Weber R T 2010 Quantitative EPR (Wien: Springer-Verlag) Online: https://www.springer.com/de/book/9783211929476

Garcia T, Lacornerie T, Popoff R, Lourenço V and Bordy J-M 2011 Dose verification and calibration of the Cyberknife $^{\circledR}$ by EPR/alanine dosimetry Radiation Measurements 46 952-7

Gordy W, Ard W, Shields H 1955 Microwave spectroscopy of biological substances. I. Paramagnetic resonace in x-irradiated amino acids and proteins. Proc. N. A. S. 41 983-996

Gustafsson H, Lund E and Olsson S 2008 Lithium formate EPR dosimetry for verifications of planned dose distributions prior to intensity-modulated radiation therapy Physics in Medicine and Biology $\mathbf{5 3}$ 4667

Haskell E H, Hayes R B and Kenner (INVITED) G H 1998 A High Sensitivity EPR Technique for Alanine Dosimetry Radiat Prot Dosimetry 77 43-9

Harding B, Tremblay C and Cousineau D 2014 Standard errors: A review and evaluation of standard error estimators using Monte Carlo simulations The Quantitative Methods for Psychology 10 107-123 doi: 10.20982/tqmp.10.2.p107

Helt-Hansen J, Rosendal F, Kofoed I M and Andersen C E 2009 Medical reference dosimetry using EPR measurements of alanine: Development of an improved method for clinical dose levels. Acta Oncologica 48 216-22

Ikeya M 1993 New Applications of Electron Spin Resonance: Dating, Dosimetry and Microscopy (World Scientific)

International organization for standardization/American society for testing and materials (ISO/ASTM 51607) (2013). Standard practice for use of the alanine-EPR dosimetry system

Komaguchi K, Matsubara Y, Shiotáni M, Gustafsson H, Lund E and Lund A 2007 An ESR and ENDOR study of irradiated 6Li-formate Spectrochimica Acta Part A: Molecular and Biomolecular Spectroscopy $66754-60$

Krivokapić A, Sanderud A, Aalbergsjø S G, Hole E O and Sagstuen E 2015 Lithium Formate for EPR Dosimetry (2): Secondary Radicals in X-Irradiated Crystals Radiation Research 183 675-83 
Lund A, Olsson S, Bonora M, Lund E and Gustafsson H 2002 New materials for ESR dosimetry Spectrochimica Acta Part A: Molecular and Biomolecular Spectroscopy 58 1301-11

Lund E, Gustafsson H, Danilczuk M, Sastry M D, Lund A, Vestad T A, Malinen E, Hole E O and Sagstuen E 2005 Formates and dithionates: sensitive EPR-dosimeter materials for radiation therapy Applied Radiation and Isotopes 62 317-24

Lund A and Shiotani M 2014 Applications of EPR in Radiation Research (Cham: Springer International Publishing) Online: http://link.springer.com/10.1007/978-3-319-09216-4

Nagy V 2000 Accuracy considerations in EPR dosimetry Applied Radiation and Isotopes 52 1039-50

Nagy V, Sleptchonok O F, Desrosiers M F, Weber R T and Heiss A H 2000a Advancements in accuracy of the alanine EPR dosimetry system: Part III: Usefulness of an adjacent reference sample Radiation Physics and Chemistry 59 429-41

Nagy V, Sholom S V, Chumak V V and Desrosiers M F 2002 Uncertainties in alanine dosimetry in the therapeutic dose range Applied Radiation and Isotopes 56 917-29

Mclaughlin W L and Desrosiers M F 1995 Dosimetry systems for radiation processing Radiation Physics and Chemistry 46 1163-74

Regulla D F and Deffner U 1982 Dosimetry by ESR spectroscopy of alanine The International Journal of Applied Radiation and Isotopes 33 1101-14

Schaeken B, Lelie S, Meijnders P, Weyngaert D V den, Janssens H and Verellen D 2010 Alanine/EPR dosimetry applied to the verification of a total body irradiation protocol and treatment planning dose calculation using a humanoid phantom Medical Physics 37 6292-9

Schaeken B, Cuypers R, Lelie S, Schroeyers W, Schreurs S, Janssens H and Verellen D 2011 Implementation of alanine/EPR as transfer dosimetry system in a radiotherapy audit programme in Belgium Radiotherapy and Oncology 99 94-6

Secerov B, Radenkovic M and Dramicanin M 2016 Uncertainty and routine use of Aerial I-alanine Electron spin resonance dosimetry system Radiation Measurements 89 63-7

Sharpe P H G, Rajendran K and Sephton J P 1996 Progress towards an alanine/ESR therapy level reference dosimetry service at NPL Applied Radiation and Isotopes 47 1171-5

Vega Ramirez J L, Chen F, Nicolucci P and Baffa O 2011 Dosimetry of small radiation field in inhomogeneous medium using alanine/EPR minidosimeters and PENELOPE Monte Carlo simulation Radiation Measurements 46 941-4

Vestad T A, Malinen E, Lund A, Hole E O and Sagstuen E 2003 EPR dosimetric properties of formates Applied Radiation and Isotopes 59 181-8 
Vestad T A, Malinen E, Olsen D R, Hole E O and Sagstuen E 2004 Electron paramagnetic resonance (EPR) dosimetry using lithium formate in radiotherapy: comparison with thermoluminescence (TL) dosimetry using lithium fluoride rods Physics in Medicine and Biology 494701

Wagner D, Anton M, Vorwerk H, Gsänger T, Christiansen H, Poppe B, Hess C F and Hermann R M 2008 In vivo alanine/electron spin resonance (ESR) dosimetry in radiotherapy of prostate cancer: a feasibility study Radiother Oncol 88 140-7

Wagner D M, Hüttenrauch P, Anton M, von Voigts-Rhetz P, Zink K and Wolff H A 2017 Feasibility study of entrance and exit dose measurements at the contra lateral breast with alanine/electron spin resonance dosimetry in volumetric modulated radiotherapy of breast cancer Physics in Medicine and Biology 62 5462-72

Waldeland E, Hörling M, Hole E O, Sagstuen E and Malinen E 2010 Dosimetry of stereotactic radiosurgery using lithium formate EPR dosimeters Phys Med Biol 55 2307-16

Waldeland E and Malinen E 2011 Review of the dose-to-water energy dependence of alanine and lithium formate EPR dosimeters and LiF TL-dosimeters - Comparison with Monte Carlo simulations Radiation Measurements 46 945-51

Waldeland E, Helt-Hansen J and Malinen E 2011 Characterization of lithium formate EPR dosimeters for high dose applications - Comparison with alanine Radiation Measurements 46 213-8

Weil J A and Bolton J R 2007 Electron Paramagnetic Resonance: Elementary Theory and Practical Applications (John Wiley \& Sons)

Wieser A and Regulla D F 1989 ESR dosimetry in the "Gigarad" range International Journal of Radiation Applications and Instrumentation. Part A. Applied Radiation and Isotopes 40 911-3

Wieser A, Lettau C, Fill U and Regulla D F 1993 The influence of non-radiation induced ESR background signal from paraffin-alanine probes for dosimetry in the radiotherapy dose range Applied Radiation and Isotopes 44 59-65 